PROCEEDINGS OF THE

AMERICAN MATHEMATICAL SOCIETY

Volume 130, Number 2, Pages 577-578

S 0002-9939(01)06070-1

Article electronically published on May 7, 2001

\title{
A NOTE ON THE COHOMOLOGICAL BELOW 1/4-PINCHING THEOREM
}

\author{
ZIZHOU TANG
}

(Communicated by Wolfgang Ziller)

\begin{abstract}
By making use of a theorem of Toda, we establish a sharper version of the below 1/4-pinching theorem of Abresch and Meyer.
\end{abstract}

By a $\delta$-pinched manifold, we mean a complete Riemannian manifold with sectional curvature $0<\delta \leq K \leq 1$. The celebrated Berger's Rigidity Theorem (see e.g. AM2 ) deals with the 1/4-pinching problem. As for the case of $\delta<1 / 4$, Abresch and Meyer AM1] proved the following result.

Theorem ([AM1 AM2]). There exists a constant $\delta_{e v} \in\left(0, \frac{1}{4}\right)$, such that, for any even-dimensional, compact, simply connected Riemannian manifold $M^{n}$ with $\delta_{\text {ev }}$-pinched sectional curvature, the cohomology rings $H^{*}\left(M^{n} ; F\right)$ with coefficients $F \in\left\{Q, Z_{2}\right\}$ are isomorphic to the corresponding cohomology rings of one of the compact, rank-one, symmetric spaces $S^{n}, C P^{n / 2}, H P^{n / 4}$, or $C a P^{2}$, or to the rings $H^{*}\left(M^{n} ; F\right)$ which are truncated polynomial rings generated by an element of degree 8.

Recall that $H^{*}\left(C a P^{2} ; F\right)=F[\xi] /\left(\xi^{3}\right)$, where $\operatorname{deg} \xi=8$. Then, it is a natural problem to decide whether there exists a manifold $M^{n}$ such that $H^{*}(M ; F)=$ $F[\xi] /\left(\xi^{m+1}\right)$, where $\operatorname{deg} \xi=8$ and $m>2$.

The purpose of this note is to point out that the exceptional alternative "or the rings $H^{n}\left(M^{n} ; F\right)$ which are truncated polynomial rings generated by an element of degree 8" can be eliminated due to the following Theorem of Toda.

Theorem $([\mathrm{To}])$. If a simply connected space $X$ has the truncated polynomial ring $Z_{p}[\xi] /\left(\xi^{m+1}\right)$ as its $\bmod p$ cohomology ring with $p=2$ or 3 and $m>2$, then $\operatorname{deg} \xi=2$ or 4 .

It should be pointed out that the condition of simple-connectivity in the preceding theorem can be eliminated [Hu, p. 304] by using $K$-theory $K(X) \otimes Z_{(2)}$ and the Adams operations.

Received by the editors October 14, 1999 and, in revised form, June 19, 2000

2000 Mathematics Subject Classification. Primary 53C20; Secondary 57R19.

Key words and phrases. Pinching constant, rank-one symmetric spaces, cohomology rings.

The author's research was partially supported by the Hong Kong Qiu-Shi Foundation (1998), the Outstanding Youth Foundation of NSF in China (No.19925103) and the Education Foundation of Tsinghua University, as well as the Grants-in-Aid for Science Research of the Japanese Ministry of Education (No.09440039). 


\section{ACKNOWLEDGEMENTS}

It is with great pleasure that I express my gratitude to the referee for useful comments and to Professors R. Miyaoka and Y. Ohnita for their invitation to visit Sophia University and TMU in Tokyo.

\section{REFERENCES}

[AM1] U. Abresch and W.T. Meyer, A sphere theorem with a pinching constant below 1/4, J. Diff. Geom. 44(1996), 214-261. MR 97i:53036

[AM2] U. Abresch and W.T. Meyer, Injectivity radius estimates and sphere theorems, In Comparison geometry, MSRI Pub. Cambridge University Press, 1997. MR 98e:53052

[Hu] D. Husemoller, Fibre bundles, GTM Vol.20, Springer-Verlag, New York, 1975. MR 51:6805

[To] H. Toda, Note on cohomology ring of certain spaces, Proc. Amer. Math. Soc. 14(1963), 89-95. MR 27:750

Department of Mathematical Sciences, Tsinghua University, Beijing, 100084, PeoPLE'S REPUBlic OF CHINA

E-mail address: zztang@mx.cei.gov.cn 